\title{
SELETIVIDADE E EICIÊNCIA DE HERBICIDAS EM CEREAIS DE INVERNO
}

\author{
Leandro Vargas ${ }^{1}$; Erivelton Scherer Roman ${ }^{2}$
}

'Eng.-Agr., D. Sc., Pesquisador da Embrapa Trigo, Caixa Postal 451, CEP 99001-970 Passo Fundo, RS.
vargas@cnpt.embrapa.br
${ }^{2}$ Eng.-Agr., Ph. D., Pesquisador da Embrapa Trigo, Caixa Postal 451, CEP 99001-970 Passo Fundo, RS.
eroman@cnpt.embrapa.br

\section{RESUMO}

O objetivo deste trabalho foi avaliar a eficiência e a seletividade de herbicidas em cereais de inverno. O experimento foi conduzido em campo, na Embrapa Trigo, no município de Passo Fundo, RS, no ano de 2005. O solo é um Latossolo Vermelho Distrófico típico, de textura média. As culturas avaliadas foram trigo, cevada, centeio e triticale. Foram semeadas na área aveia-preta, aveia-branca, nabo e ervilhaca, espécies que constituiram-se nas plantas daninhas avaliadas. $O$ herbicidas avaliados foram bentazon, metsulfuron-methyl, diclofop-methyl, clodinafop-propargil, 2,4-D, 2,4-D + picloram, 2,4-D + glifosato (aplicação sequencial) e iodosulfurommetílico. Os tratamentos herbicidas pré-emergentes foram aplicados no dia 23/06/2005, um dia antes da semeadura das culturas. Os pós-emergentes no dia 27/07/05, quando as espécies avaliadas estavam no estádio de 3-5 folhas. Os resultados, de forma geral, indicam que os herbicidas, nas doses avaliadas, são seletivos para as culturas do trigo, triticale e centeio, e que a cevada apresenta maior sensibilidade aos herbicidas, principalmente ao iodosulfurom-metílico, indicando que a aplicação deste herbicida deve ser criteriosa. Evidenciam ainda que a aveia-preta, a aveia-branca e o azevém apresentam resposta diferenciada aos herbicidas diclofop-methyl, clodinafop-propargil e iodosulfurom-metílico, sendo que a dose é fator importante no nível de controle de cada espécie, devendo-se proceder recomendações específicas para cada uma delas. Já o nabo e a ervilhaca são controlados com eficiência pelos herbicidas iodosulfurom-metílico e 2,4-D e 2,4-D + picloram.

Palavras-chave: trigo, plantas daninhas, fitotoxicidade.

\section{ABSTRACT \\ Selectivity and efficacy of herbicides to winter cereals}

An experiment was carried-out with the objective of evaluating the selectivity and efficacy of herbicides applied for weed control on winter cereals. The experiment was conducted under field conditions at the field station of Embrapa, in Passo Fundo, RS, during the 2005 growing season. The soil of the station is a loam Oxisol. The evaluated crops were wheat, barley, rye and triticale. Black oats, oats, turnipseed and vetch were sown in order to act as volunteer crops interfering with those crops. The tested herbicides were bentazon, metsulfuron-methyl, dichlofop-methyl, 
clodinafop-propargyl, 2,4-D, 2,4-D + picloran, 2,4-D + glyphosate (as sequential application) and iodosulfuron-methyl. The pre-emergence treatments were applied in $23 / 06 / 2005$, one day before sowing the crops. The post-emergence treatments were applied in 27/07/2005 at the 3-5 leave weed stage. The results indicated that the treatments were selective to wheat, triticale and rye while barley was the species more sensible to them especially to iodosulfuron-methyl. Black oats, oats, and ryegrass responded differently to the treatments according to the tested rate while turnipseed and vetch are efficiently controlled by iodosulfuron-methyl, 2,4-D and 2,4$\mathrm{D}+$ picloram.

Key-words: wheat, weeds, phytotoxicity.

\section{INTRODUÇÃO}

Os cereais de inverno - trigo, centeio, cevada e triticale - são cultivados principalmente na região sul do Brasil. O trigo é cultivado em diversos estados brasileiros, e são várias as espécies daninhas que causam perdas econômicas na produtividade dessa cultura. $\mathrm{Na}$ região sul do Brasil, as gramíneas Lolium multiflorum (azevém), Avena strigosa (aveia preta) e Avena sativa (aveia branca) causam os maiores prejuízos em cereais. Entre as dicotiledôneas destacam-se: Raphanus raphanistrum e Raphanus sativus (nabo ou nabiça), Polygonum convolvulus (cipó de veado ou erva de bicho), Rumex spp. (língua de vaca), Echium plantagineum (flor roxa), Bowlesia incana (erva salsa), Sonchus oleraceus (serralha), Silene gallica (silene), Spergula arvensis (gorga ou espérgula) e Stellaria media (esparguta). Em anos em que o inverno apresenta temperatura média elevada, ocorrem também outras invasoras de folhas largas, mais comuns no verão, como Bidens pilosa (picão preto), Ipomoea spp. (corriola) e Richardia brasiliensis (poaia).

Azevém e aveia-preta tiveram as respectivas ocorrências e densidades aumentadas nos últimos anos, em virtude de seu uso em cobertura de solo e em rotação de culturas e da conseqüente ressemeadura natural.

Estimativas de perdas na produtividade de cereais de inverno são imprecisas. O grau de competição das plantas daninhas varia de acordo com as espécies infestantes, com a densidade populacional, com a duração da competição e com as condições de ambiente (Swanton \& Weise, 1991).

Perdas causadas por plantas daninhas na produtividade podem estar associadas à competição, pelo efeito da alelopatia ou, indiretamente, à redução da qualidade do produto colhido. A competição ocorre quando qualquer fator de ambiente (água, luz ou nutrientes) é limitado e dividido entre a cultura e as invasoras e se torna restritivo à obtenção de produtividades elevadas. A alelopatia é a inibição do crescimento de uma planta por outra, através da liberação de compostos químicos no ambiente.

A redução mais acentuada da produtividade em trigo verifica-se quando a competição ocorre nos estádios iniciais de desenvolvimento da cultura (denominado período crítico de competição), que se estende até 45 a 50 dias após a emergência de plantas (Blanco et al., 1973). De modo geral, as culturas devem permanecer livres de competição no primeiro terço de seu desenvolvimento. Nesse contexto, o período crítico de uma cultivar com ciclo de 140 dias terminaria aos 47 dias após a emergência. No entanto, esse período pode variar em função das condições de ambiente que afetam o crescimento das espécies em competição. Durante esse período, os prejuízos provocados são irreversíveis, e é por isso que nessa época o 
trigo deve estar livre da interferência de plantas daninhas.

Embora a competição tardia não afete substancialmente o rendimento de grãos, ela pode interferir nas operações de colheita e na qualidade do produto colhido. A contaminação de grãos com partes de plantas daninhas, e/ou com sementes destas, provoca depreciação do produto. As sementes de erva-de-bicho (Polygonum spp.), por exemplo, têm sabor amargo, o que pode alterar a qualidade da farinha. Além de dificultarem a colheita, as plantas daninhas podem elevar a umidade dos grãos e os custos de secagem, favorecer a fermentação, aumentar a incidência de pragas no armazenamento e, até mesmo, diminuir o valor recebido pelos produtores, em razão de descontos gerados por impureza e umidade de grãos. Dessa forma, o controle adequado de plantas daninhas na cultura de trigo com produtos seletivos e seguros, tanto para a cultura como para o ambiente, é extremamente importante. Assim, o objetivo deste trabalho foi avaliar a eficiência e a seletividade de herbicidas em cereais de inverno.

\section{MATERIAL E MÉTODOS}

O experimento foi conduzido em condições de campo na área experimental da Embrapa Trigo, localizada no município de Passo Fundo, RS, no ano de 2005. O solo é de textura média, com $42,0 \%$ de argila e $4,0 \%$ de matéria orgânica, pertencente à Unidade de Mapeamento Passo Fundo (Latossolo Vermelho distrófico típico). A semeadura dos cereais foi realizada em 24 de junho de 2005, em sistema plantio direto, com espaçamento entre as linhas de $0,17 \mathrm{~m}$, densidade de 60 sementes por metro linear. A adubação foi realizada usando-se $250 \mathrm{~kg} \mathrm{ha}^{-1}$ de adubo, fórmula 5-25-25. Os tratamentos herbicidas avaliados constam na Tabela 1. As culturas avaliadas foram trigo (cultivar BRS 194), cevada (cultivar BRS 225), centeio (cultivar BR 1) e triticale (cultivar BRS 148). Foram semeados na área aveia preta, aveia branca, nabo e ervilhaca, espécies que constituíram-se nas plantas daninhas avaliadas. Os tratamentos herbicidas pré-emergentes foram aplicados em 23/6/2005, um dia antes da semeadura das culturas; os pós-emergentes, no dia 27/7/05, quando as espécies avaliadas estavam no estádio de 3-5 folhas. A aplicação dos tratamentos foi realizada usando-se pulverizador costal de precisão, com pressão de trabalho de $15 \mathrm{lb} . /$ pol. $^{-2}$, dada por gás carbônico, munido de bicos de jato em forma de leque, tipo 110015 , espaçados $0,5 \mathrm{~m}$ entre si e posicionados à altura de $50 \mathrm{~cm}$ acima do alvo. O volume de calda usado foi de $100 \mathrm{~L} \mathrm{ha}^{-1}$. O delineamento experimental foi de blocos ao acaso, com 4 repetições. As parcelas mediam $3 \mathrm{~m} \times 4 \mathrm{~m}$.

Tabela 1. Tratamentos, doses e época de aplicação. Embrapa Trigo-CNTP, Passo Fundo, RS, 2005.

\begin{tabular}{|c|c|c|c|c|c|}
\hline \multirow{2}{*}{$\mathbf{N}^{\circ}$} & \multicolumn{2}{|c|}{ HERBICIDA } & $\begin{array}{c}\text { Produto } \\
\text { comercial } \\
\left.\text { (L ou g ha }{ }^{-1}\right)\end{array}$ & $\begin{array}{c}\text { Ativo } \\
\left(\mathbf{g} \text { i. a. ha } \mathbf{~}^{-1} \text { ) }\right.\end{array}$ & $\begin{array}{c}\text { Época } \\
\text { aplica- } \\
\text { ção }\end{array}$ \\
\cline { 2 - 6 } & \multicolumn{2}{|c|}{ Nome comercial } & Nome comum & 720 & Pós \\
\hline 1 & Basagram & bentazon & 1,5 & 720 \\
\hline
\end{tabular}




\begin{tabular}{|c|c|c|c|c|c|}
\hline 2 & Basagran & bentazon & 2,5 & 1200 & Pós \\
\hline 3 & Ally & metsulfuron-methyl & 4 & 2,4 & Pós \\
\hline 4 & Ally & metsulfuron-methyl & 6 & 3,6 & Pós \\
\hline 5 & lloxan & diclofop-methyl & 1,5 & 426 & Pós \\
\hline 6 & lloxan & diclofop-methyl & 3,0 & 852 & Pós \\
\hline 7 & Topik & clodinafop-propargil & 100 & 24 & Pós \\
\hline 8 & Topik & clodinafop-propargil & 200 & 48 & Pós \\
\hline 9 & Herbi D480 & $2,4-D$ amina & 1 & 480 & Pós \\
\hline 10 & Herbi D480 & $2,4-D$ amina & 2 & 960 & Pós \\
\hline 11 & Dontor & $2,4-D+$ picloran & 1,5 & $540+$ & Pós \\
\hline 12 & Dontor & $2,4-D+$ picloran & & 33,75 & \\
\hline 13 & Herbi D 480+ Trop* & 2,4-D amina + glifosato & $1,5+2$ & $720+720$ & Pré \\
\hline 14 & Herbi D 480+ Trop* & 2,4-D amina + glifosato & $2+2$ & $960+720$ & Pré \\
\hline 15 & Hussar & lodosulfurom-metílico & 70 & 3,5 & Pós \\
\hline 16 & Hussar & lodosulfurom-metílico & 100 & 57,5 & Pós \\
\hline 17 & Testemunha & -- & - & -- & - - \\
\hline
\end{tabular}

* Tratamento aplicado de forma seqüencial.

A seletividade e a eficiência dos tratamentos foram determinadas aos 7, 14, 25 e 40 dias após a aplicação (DAA), pelo método de avaliação visual, atribuindo-se notas em porcentagem de controle em relação à testemunha. A escala empregada variou entre 0 (sem danos visíveis) e 100\% (morte das plantas). A avaliação de seletividade às culturas foi realizada visualmente, e os resultados expressos em porcentagem de danos, tomando como base a redução do crescimento, a clorose e a necrose foliar.

Os dados de seletividade e eficiência dos tratamentos foram analisados pela variância, e as médias comparadas, pelo teste de Duncan, a 5\% de probabilidade de erro.

\section{RESULTADOS E DISCUSSÃO}

$\mathrm{Na}$ avaliação da seletividade dos tratamentos herbicidas, não foram observadas sintomas de fitotoxicidade nas culturas de trigo, de triticale e de centeio. Entretanto, a cevada manifestou sintomas, caracterizados por clorose das folhas (folhas amareladas), em resposta aos tratamentos herbicidas metsulfuron-methyl, clodinafop-propargil e iodosulfurom-metílico (Tabela 2). 
Tabela 2. Avaliação da fitotoxicidade em cevada em resposta à aplicação de diferentes herbicidas e doses. Embrapa Trigo-CNPT, Passo Fundo, RS, 2005.

\begin{tabular}{|c|c|c|c|c|c|c|}
\hline $\mathbf{N}^{\mathbf{0}}$ & Herbicida & $\begin{array}{c}\text { Produto } \\
\text { comercial } \\
\text { (L ou g ha }^{-1} \text { ) }\end{array}$ & 7 DAT & $\mathbf{1 4}$ DAT & 25 DAT & 40 DAT \\
\hline 1 & bentazon & 1,5 & $0 \mathrm{~d}$ & $0 \mathrm{c}$ & $0 \mathrm{c}$ & $0 \mathrm{~b}$ \\
\hline 2 & bentazon & 2,5 & $0 \mathrm{~d}$ & $0 \mathrm{c}$ & $0 \mathrm{c}$ & $0 \mathrm{~b}$ \\
\hline 3 & metsulfuron-methyl & 4 & $0 \mathrm{~d}$ & $0 \mathrm{c}$ & $0 \mathrm{c}$ & $0 \mathrm{~b}$ \\
\hline 4 & metsulfuron-methyl & 6 & $8 \mathrm{c}$ & $15 \mathrm{~b}$ & $5 \mathrm{~b}$ & $0 \mathrm{~b}$ \\
\hline 5 & diclofop-methyl & 1,5 & $0 \mathrm{~d}$ & $0 \mathrm{c}$ & $0 \mathrm{c}$ & $0 \mathrm{~b}$ \\
\hline 6 & diclofop-methyl & 3,0 & $0 \mathrm{~d}$ & $0 \mathrm{c}$ & $0 \mathrm{c}$ & $0 \mathrm{~b}$ \\
\hline 7 & clodinafop-propargil & 100 & $0 \mathrm{~d}$ & $0 \mathrm{c}$ & $0 \mathrm{c}$ & $0 \mathrm{~b}$ \\
\hline 8 & clodinafop-propargil & 200 & $20 \mathrm{~b}$ & $15 \mathrm{~b}$ & $5 \mathrm{~b}$ & $0 \mathrm{~b}$ \\
\hline 9 & $2,4-D$ amina & 1,0 & $0 \mathrm{~d}$ & $0 \mathrm{c}$ & $0 \mathrm{c}$ & $0 \mathrm{~b}$ \\
\hline 10 & $2,4-D$ amina & 2,0 & $0 \mathrm{~d}$ & $0 \mathrm{c}$ & $0 \mathrm{c}$ & $0 \mathrm{~b}$ \\
\hline 11 & $2,4-D+$ picloran & 1,5 & $0 \mathrm{~d}$ & $0 \mathrm{c}$ & $0 \mathrm{c}$ & $0 \mathrm{~b}$ \\
\hline 12 & $2,4-D+$ picloran & 3,0 & $0 \mathrm{~d}$ & $0 \mathrm{c}$ & $0 \mathrm{c}$ & $0 \mathrm{~b}$ \\
\hline 13 & $2,4-D$ amina + glifosato & $1,5+2,0$ & $0 \mathrm{~d}$ & $0 \mathrm{c}$ & $0 \mathrm{c}$ & $0 \mathrm{~b}$ \\
\hline 14 & $2,4-D$ amina + glifosato & $2,0+2,0$ & $0 \mathrm{~d}$ & $0 \mathrm{c}$ & $0 \mathrm{c}$ & $0 \mathrm{~b}$ \\
\hline 15 & lodosulfurom-metílico & 70 & $0 \mathrm{~d}$ & $0 \mathrm{c}$ & $0 \mathrm{c}$ & $0 \mathrm{~b}$ \\
\hline 16 & lodosulfurom-metílico & 100 & $36 \mathrm{a}$ & $30 \mathrm{a}$ & $25 \mathrm{a}$ & $20 \mathrm{a}$ \\
\hline 17 & Testemunha & -- & $0 \mathrm{~d}$ & $0 \mathrm{c}$ & $0 \mathrm{c}$ & $0 \mathrm{~b}$ \\
\hline
\end{tabular}

Médias seguidas pela mesma letra, na coluna, não diferem significativamente, pelo teste de Duncan, ao nível de $5 \%$ de probabilidade.

A fitotoxicidade, aos 7 DAT, foi maior no tratamento com o herbicida iodosulfurom-metílico, $36 \%$, na dose de $100 \mathrm{~g}$ p.c. ha ${ }^{-1}$. Já o tratamento com clodinafop-propargil, na dose de $200 \mathrm{~mL}$ p.c. ha ${ }^{-1}$, apresentou fitotoxicidade em intensidade intermediária. O herbicida metsulfuron-methyl, na dose de $6 \mathrm{~g}$ p.c. ha ${ }^{-1}$, provocou toxicidade de $5 \%$ (Tabela 2). Não foram observados sintomas de fitotoxicidade ocasionados pelos demais tratamentos.

$\mathrm{Na}$ avaliação realizada aos 14 DAT, observou-se que os sintomas de fitotoxicidade, de forma geral, diminuíram. Contudo, o herbicida iodosulfurommetílico, na dose de $100 \mathrm{~g}$ p.c. ha ${ }^{-1}$, continuou evidenciando $30 \%$ de fitotoxicidade, superior ao verificado nos demais tratamentos herbicidas. Os tratamentos com clodinafop-propargil e metsulfuron-methyl apresentaram fitotoxicidade semelhante, em torno de $15 \%$. Para os demais tratamentos, não foram observados sintomas de fitotoxicidade.

Aos 25 DAT, observou-se $5 \%$ de fitotoxicidade nos tratamentos com clodinafop-propargil e com metsulfuron-methyl e de $25 \%$ para o iodosulfurommetílico (Tabela 2). Na última avaliação, realizada aos 40 DAT, observou-se que os sintomas de fitotoxicidade foram superados pela cultura de cevada, e somente o tratamento com $100 \mathrm{~g}$ p.c. ha ${ }^{-1}$ de iodosulfurom-metílico apresentou sintomas de toxicidade, com estimativa de $20 \%$ de danos (Tabela 2 ).

Não foram observados efeitos significativos dos tratamentos herbicidas avaliados sobre a população e a altura de plantas de trigo, de cevada, de triticale e de centeio. Isso evidencia que os herbicidas testados não afetam a germinação e o crescimento de plantas dessas culturas, não obstante os sintomas de fitotoxicidade, caracterizados por intensa clorose, principalmente em cevada, em resposta ao herbicida iodosulfurom-metílico. Vale salientar que a dose recomendada desse herbicida para a cultura de cevada é de $70 \mathrm{~g}$ p.c. ha ${ }^{-1}$ e que, no tratamento com essa dose, não se observaram sintomas expressivos de fitotoxicidade. Destaca-se que o mesmo ocorreu com os herbicidas clodinafop-propargil e metsulfuron-methyl, em 
que observaram-se sintomas de fitoxicidade nas maiores doses avaliadas, ou seja, essas doses não são recomendadas para a cultura de cevada e foram aplicadas neste experimento para avaliação da seletividade e segurança desses produtos em função da dose empregada.

Trigo e cevada apresentam elevada tolerância a diclofop-methyl (GarciaTorres \& Fernandes-Quintanilla, 1991), e, dessa forma, era esperado que o centeio e, principalmente, o triticale não evidenciassem sintomas de fitotoxicidade resultante do tratamento com diclofop.

Os dados de controle das espécies daninhas aveia preta, aveia branca, azevém, nabo e ervilhaca, aos 7 DAT, constam na Tabela 3. Nessa avaliação, o herbicida clodinafop-propargil, na dose de $200 \mathrm{~mL}$ p.c. ha ${ }^{-1}$, apresentou o maior controle de aveia preta (60\%), de aveia branca (55\%) e de azevém (60\%). A dose de $100 \mathrm{~mL}$ p.c. ha $^{-1}$ de clodinafop-propargil apresentou controles de $45 \%$ de aveia preta, $40 \%$ de aveia branca e de $45 \%$ do azevém, os quais não diferiram do controle obtido com as maiores doses avaliadas dos herbicidas iodosulfurom-metílico e diclofop-methyl. Os demais tratamentos não proporcionaram nenhum controle dessas gramíneas.

Tabela 3. Avaliação da fitotoxicidade, aos 7 DAT, em aveia branca, em aveia preta, em nabo e em ervilhaca, em resposta à aplicação de diferentes herbicidas e doses. Embrapa Trigo-CNPT, Passo Fundo, RS, 2005.

\begin{tabular}{|c|c|c|r|r|r|r|r|}
\hline $\mathbf{N}^{\circ}$ & Herbicida & $\begin{array}{c}\text { Produto } \\
\text { comercial } \\
\left(\begin{array}{c}\text { L ou g ha } \\
\text {-1 }\end{array}\right.\end{array}$ & $\begin{array}{c}\text { Aveia } \\
\text { preta }\end{array}$ & $\begin{array}{c}\text { Aveia } \\
\text { branca }\end{array}$ & Azevém & Nabo & Ervilhaca \\
\hline 1 & bentazon & 1,5 & $0 \mathrm{~d}$ & $0 \mathrm{~d}$ & $0 \mathrm{c}$ & $30 \mathrm{~d}$ & $25 \mathrm{~d}$ \\
\hline 2 & bentazon & 2,5 & $0 \mathrm{~d}$ & $0 \mathrm{~d}$ & $0 \mathrm{c}$ & $40 \mathrm{~d}$ & $30 \mathrm{~d}$ \\
\hline 3 & metsulfuron-methyl & 4 & $0 \mathrm{~d}$ & $0 \mathrm{~d}$ & $0 \mathrm{c}$ & $65 \mathrm{c}$ & $30 \mathrm{~d}$ \\
\hline 4 & metsulfuron-methyl & 6 & $0 \mathrm{~d}$ & $0 \mathrm{~d}$ & $0 \mathrm{c}$ & $80 \mathrm{ab}$ & $45 \mathrm{c}$ \\
\hline 5 & diclofop-methyl & 1,5 & $30 \mathrm{c}$ & $25 \mathrm{c}$ & $35 \mathrm{~b}$ & $0 \mathrm{e}$ & $0 \mathrm{f}$ \\
\hline 6 & diclofop-methyl & 3,0 & $40 \mathrm{bc}$ & $30 \mathrm{bc}$ & $45 \mathrm{~b}$ & $0 \mathrm{e}$ & $0 \mathrm{f}$ \\
\hline 7 & clodinafop-propargil & 100 & $45 \mathrm{~b}$ & $40 \mathrm{~b}$ & $45 \mathrm{~b}$ & $0 \mathrm{e}$ & $0 \mathrm{f}$ \\
\hline 8 & clodinafop-propargil & 200 & $60 \mathrm{a}$ & $55 \mathrm{a}$ & $60 \mathrm{a}$ & $0 \mathrm{e}$ & $0 \mathrm{f}$ \\
\hline 9 & $2,4-D$ amina & 1,0 & $0 \mathrm{~d}$ & $0 \mathrm{~d}$ & $0 \mathrm{c}$ & $70 \mathrm{bc}$ & $90 \mathrm{a}$ \\
\hline 10 & $2,4-D$ amina & 2,0 & $0 \mathrm{~d}$ & $0 \mathrm{~d}$ & $0 \mathrm{c}$ & $85 \mathrm{ab}$ & $95 \mathrm{a}$ \\
\hline 11 & $2,4-D+$ picloran & 1,5 & $0 \mathrm{~d}$ & $0 \mathrm{~d}$ & $0 \mathrm{c}$ & $90 \mathrm{a}$ & $95 \mathrm{a}$ \\
\hline 12 & $2,4-D+$ picloran & 3,0 & $0 \mathrm{~d}$ & $0 \mathrm{~d}$ & $0 \mathrm{c}$ & $95 \mathrm{a}$ & $95 \mathrm{a}$ \\
\hline 13 & $2,4-D$ amina + glifosato & $1,5+2,0$ & $0 \mathrm{~d}$ & $0 \mathrm{~d}$ & $0 \mathrm{c}$ & $75 \mathrm{bc}$ & $10 \mathrm{ef}$ \\
\hline 14 & $2,4-D$ amina + glifosato & $2,0+2,0$ & $0 \mathrm{~d}$ & $0 \mathrm{~d}$ & $0 \mathrm{c}$ & $80 \mathrm{ab}$ & $20 \mathrm{de}$ \\
\hline 15 & lodosulfurom-metílico & 70 & $35 \mathrm{bc}$ & $26 \mathrm{c}$ & $43 \mathrm{~b}$ & $80 \mathrm{ab}$ & $60 \mathrm{~b}$ \\
\hline 16 & lodosulfurom-metílico & 100 & $40 \mathrm{bc}$ & $35 \mathrm{bc}$ & $45 \mathrm{~b}$ & $90 \mathrm{a}$ & $70 \mathrm{~b}$ \\
\hline 17 & Testemunha & -- & $0 \mathrm{~d}$ & $0 \mathrm{~d}$ & $0 \mathrm{c}$ & $0 \mathrm{e}$ & $0 \mathrm{f}$ \\
\hline
\end{tabular}

Médias seguidas pela mesma letra, na coluna, não diferem significativamente, pelo teste de Duncan, ao nível de $5 \%$ de probabilidade.

O clodinafop-propargil e diclofop-methyl não apresentaram nenhum efeito sobre o nabo e a ervilhaca. Já iodosulfurom-metílico evidenciou o maior controle de nabo (90\%), com a dose de $100 \mathrm{~g}$ p.c. $\mathrm{ha}^{-1}$, semelhante àquele proporcionado pela dose de $70 \mathrm{~g}$ p.c. ha ${ }^{-1}$ desse herbicida e pelos herbicidas contendo 2,4-D, na dose de $2 \mathrm{~L}$ p.c. ha ${ }^{-1}$, aplicados em pré e pós-emergência, e 2,4-D mais picloram (Tabela 3). A ervilhaca apresentou-se mais sensível aos herbicidas contendo 2,4-D e 2,4-D mais picloram, quando aplicados na pós-emergência (Tabela 3). Os herbicidas 2,4$D$, aplicado na pré-emergência de forma sequencial ao glyphosate na dessecação, 
metsulfuron-methyl e bentazon apresentaram baixo controle de ervilhaca (10 a $45 \%)$.

$\mathrm{Na}$ avaliação realizada aos 14 DAT (Tabela 4), o herbicida clodinafoppropargil, na dose de $200 \mathrm{~mL}$ p.c. ha ${ }^{-1}$, apresentou o maior controle de aveia preta $(90 \%)$ e de aveia branca (85\%), semelhante aos controles obtidos com a dose de $100 \mathrm{~mL}$ p.c. ha ${ }^{-1}$ desse herbicida e com o da maior dose avaliada de iodosulfurommetílico. Os demais tratamentos herbicidas contendo clodinafop-propargil, iodosulfurom-metílico e diclofop-methyl apresentaram controles intermediários de aveia preta e de aveia branca (Tabela 4). Já o azevém teve seu maior controle $(95 \%)$ no tratamento com a maior dose, $100 \mathrm{~g}$ p.c. ha ${ }^{-1}$, do herbicida iodosulfurommetílico. Os demais tratamentos novamente não proporcionaram nenhum controle das espécies daninhas gramíneas.

Os tratamentos herbicidas com 2,4-D, aplicado em pós-emergência, e 2,4-D mais picloram controlaram totalmente 0 nabo nesta avaliação. $O$ herbicida iodosulfurom-metílico, na dose de $100 \mathrm{~g}$ p.c. ha ${ }^{-1}$, evidenciou controle de $95 \%$ de nabo, semelhante àquele obtido nos tratamentos com 2,4-D. A ervilhaca foi controlada com eficiência pelos tratamentos contendo 2,4-D, aplicados em pósemergência, 2,4-D mais picloram e iodosulfurom-metílico, em ambas as doses avaliadas (Tabela 4). Os herbicidas2,4-D, aplicado na pré-emergência, e metsulfuron-methyl e bentazon apresentaram baixo controle de ervilhaca (15 a $45 \%)$.

Tabela 4. Avaliação da fitotoxicidade, aos 14 DAT, em aveia branca, em aveia preta, em nabo e em ervilhaca, em resposta à aplicação de diferentes herbicidas e doses. Embrapa Trigo-CNPT, Passo Fundo, RS, 2005.

\begin{tabular}{|c|c|c|c|c|c|c|c|}
\hline $\mathbf{N}^{\circ}$ & Herbicidas & $\begin{array}{l}\text { Produto } \\
\text { comercial } \\
\text { (L ou g ha- } \\
\text { ) }\end{array}$ & $\begin{array}{l}\text { Aveia } \\
\text { preta }\end{array}$ & $\begin{array}{c}\text { Aveia } \\
\text { branca }\end{array}$ & Azevém & Nabo & Ervilhaca \\
\hline 1 & bentazon & 1,5 & $0 \mathrm{e}$ & $0 \mathrm{~d}$ & $0 \mathrm{c}$ & $45 \mathrm{e}$ & $40 \mathrm{~b}$ \\
\hline 2 & bentazon & 2,5 & $0 \mathrm{e}$ & $0 \mathrm{~d}$ & $0 \mathrm{c}$ & $50 \mathrm{e}$ & $45 \mathrm{~b}$ \\
\hline 3 & metsulfuron-methyl & 4 & $0 \mathrm{e}$ & $0 \mathrm{~d}$ & $0 \mathrm{c}$ & $70 \mathrm{~d}$ & $25 \mathrm{c}$ \\
\hline 4 & metsulfuron-methyl & 6 & $0 \mathrm{e}$ & $0 \mathrm{~d}$ & $0 \mathrm{c}$ & $80 \mathrm{~cd}$ & $40 \mathrm{~b}$ \\
\hline 5 & diclofop-methyl & 1,5 & $60 \mathrm{~d}$ & $55 \mathrm{c}$ & $75 \mathrm{~b}$ & $0 \mathrm{f}$ & $0 \mathrm{~d}$ \\
\hline 6 & diclofop-methyl & 3,0 & $75 \mathrm{bcd}$ & $65 \mathrm{bc}$ & $85 \mathrm{ab}$ & $0 \mathrm{f}$ & $0 \mathrm{~d}$ \\
\hline 7 & clodinafop-propargil & 100 & $80 \mathrm{abc}$ & $75 \mathrm{ab}$ & $75 \mathrm{~b}$ & $0 \mathrm{f}$ & $0 \mathrm{~d}$ \\
\hline 8 & clodinafop-propargil & 200 & $90 \mathrm{a}$ & $85 \mathrm{a}$ & $80 \mathrm{ab}$ & $0 \mathrm{f}$ & $0 \mathrm{~d}$ \\
\hline 9 & 2,4-D amina & 1,0 & $0 \mathrm{e}$ & $0 \mathrm{~d}$ & $0 \mathrm{c}$ & $100 \mathrm{a}$ & $90 \mathrm{a}$ \\
\hline 10 & 2,4-D amina & 2,0 & $0 \mathrm{e}$ & $0 \mathrm{~d}$ & $0 \mathrm{c}$ & $100 \mathrm{a}$ & $95 \mathrm{a}$ \\
\hline 11 & 2,4-D + picloran & 1,5 & $0 \mathrm{e}$ & $0 \mathrm{~d}$ & $0 \mathrm{c}$ & $100 \mathrm{a}$ & $95 \mathrm{a}$ \\
\hline 12 & 2,4-D + picloran & 3,0 & $0 \mathrm{e}$ & $0 \mathrm{~d}$ & $0 \mathrm{c}$ & $100 \mathrm{a}$ & $100 \mathrm{a}$ \\
\hline 13 & 2,4-D amina + glifosato & $1,5+2,0$ & $0 \mathrm{e}$ & $0 \mathrm{~d}$ & $0 \mathrm{c}$ & $80 \mathrm{~cd}$ & $15 \mathrm{c}$ \\
\hline 14 & 2,4-D amina + glifosato & $2,0+2,0$ & $0 \mathrm{e}$ & $0 \mathrm{~d}$ & $0 \mathrm{c}$ & $85 \mathrm{bc}$ & $25 \mathrm{c}$ \\
\hline 15 & lodosulfurom-metílico & 70 & $70 \mathrm{~cd}$ & $65 \mathrm{bc}$ & $80 \mathrm{ab}$ & $80 \mathrm{~cd}$ & $100 \mathrm{a}$ \\
\hline 16 & lodosulfurom-metílico & 100 & $85 \mathrm{ab}$ & $80 a$ & $95 \mathrm{a}$ & $95 \mathrm{a}$ & $100 \mathrm{a}$ \\
\hline 17 & Testemunha & -- & $0 \mathrm{e}$ & $0 \mathrm{~d}$ & $0 \mathrm{c}$ & $0 \mathrm{f}$ & $0 \mathrm{~d}$ \\
\hline
\end{tabular}

Médias seguidas pela mesma letra, na coluna, não diferem significativamente, pelo teste de Duncan, ao nível de $5 \%$ de probabilidade.

$\mathrm{Na}$ avaliação realizada aos 25 DAT (Tabela 5), o herbicida clodinafoppropargil, na dose de $200 \mathrm{~mL}^{\text {p.c. }}$ ha $^{-1}$, e o herbicida iodosulfurom-metílico, na dose de 100 g p.c. ha ${ }^{-1}$, apresentaram os maiores controles, acima de $90 \%$, de aveia preta e de aveia branca. Os demais tratamentos herbicidas contendo clodinafop- 
propargil, iodosulfurom-metílico e diclofop-methyl apresentaram controles entre 55 e $85 \%$, tanto de aveia preta como da aveia branca (Tabela 5). O melhor controle de azevém foi obtido com os tratamentos contendo diclofop-methyl ou iodosulfurommetílico, em ambas as doses avaliadas, que foi semelhante àquele obtido no

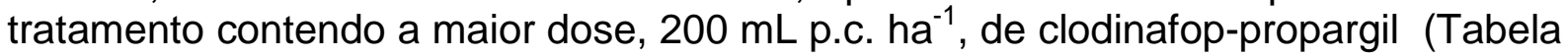
5).

Nesta avaliação, realizada aos 25 DAT, o nabo foi controlado totalmente com o herbicida 2,4-D, aplicado em pós-emergência, e 2,4-D mais picloram. Novamente o herbicida iodosulfurom-metílico, na dose de $100 \mathrm{~g}$ p.c. ha ${ }^{-1}$, evidenciou controle de nabo semelhante aos tratamentos com 2,4-D. O 2,4-D, aplicado na préemergência, proporcionou controle de nabo de 85 e 90\%, respectivamente para as dose de 1,5 e 2,0 L ha-1. A ervilhaca foi controlada com eficiência pelos tratamentos contendo 2,4-D, aplicados em pós-emergência, 2,4-D mais picloram e iodosulfurommetílico, em ambas as doses avaliadas. Os herbicidas 2,4-D, aplicado na préemergência, e metsulfuron-methyl e bentazon apresentaram baixo controle de ervilhaca (15 a 45\%).

Tabela 5. Avaliação da fitotoxicidade, aos 25 DAT, em aveia branca, em aveia preta, em nabo e em ervilhaca, em resposta à aplicação de diferentes herbicidas e doses. Embrapa Trigo-CNPT, Passo Fundo, RS, 2005.

\begin{tabular}{|r|c|r|r|r|r|r|r|}
\hline \multirow{2}{*}{$\mathbf{N}^{\mathbf{0}}$} & Herbicida & $\begin{array}{c}\text { Produto } \\
\text { comercial } \\
\left(\begin{array}{c}\text { L ou g ha } \\
\text {-1 })\end{array}\right.\end{array}$ & $\begin{array}{c}\text { Aveia } \\
\text { preta }\end{array}$ & $\begin{array}{c}\text { Aveia } \\
\text { branca }\end{array}$ & Azevém & Nabo & Ervilhaca \\
\hline 1 & bentazon & 1,5 & $0 \mathrm{e}$ & $0 \mathrm{~d}$ & $0 \mathrm{c}$ & $50 \mathrm{~d}$ & $45 \mathrm{~b}$ \\
\hline 2 & bentazon & 2,5 & $0 \mathrm{e}$ & $0 \mathrm{~d}$ & $0 \mathrm{c}$ & $55 \mathrm{~d}$ & $50 \mathrm{~b}$ \\
\hline 3 & metsulfuron-methyl & 4 & $0 \mathrm{e}$ & $0 \mathrm{~d}$ & $0 \mathrm{c}$ & $75 \mathrm{c}$ & $20 \mathrm{c}$ \\
\hline 4 & metsulfuron-methyl & 6 & $0 \mathrm{e}$ & $0 \mathrm{~d}$ & $0 \mathrm{c}$ & $80 \mathrm{bc}$ & $40 \mathrm{~b}$ \\
\hline 5 & diclofop-methyl & 1,5 & $70 \mathrm{~d}$ & $55 \mathrm{c}$ & $90 \mathrm{ab}$ & $0 \mathrm{e}$ & $0 \mathrm{~d}$ \\
\hline 6 & diclofop-methyl & 3,0 & $80 \mathrm{~cd}$ & $65 \mathrm{c}$ & $95 \mathrm{a}$ & $0 \mathrm{e}$ & $0 \mathrm{~d}$ \\
\hline 7 & clodinafop-propargil & 100 & $85 \mathrm{bc}$ & $80 \mathrm{~b}$ & $80 \mathrm{~b}$ & $0 \mathrm{e}$ & $0 \mathrm{~d}$ \\
\hline 8 & clodinafop-propargil & 200 & $98 \mathrm{a}$ & $95 \mathrm{a}$ & $95 \mathrm{a}$ & $0 \mathrm{e}$ & $0 \mathrm{~d}$ \\
\hline 9 & $2,4-D$ amina & 1,0 & $0 \mathrm{e}$ & $0 \mathrm{~d}$ & $0 \mathrm{c}$ & $100 \mathrm{a}$ & $95 \mathrm{a}$ \\
\hline 10 & $2,4-D$ amina & 2,0 & $0 \mathrm{e}$ & $0 \mathrm{~d}$ & $0 \mathrm{c}$ & $100 \mathrm{a}$ & $100 \mathrm{a}$ \\
\hline 11 & $2,4-D+$ picloran & 1,5 & $0 \mathrm{e}$ & $0 \mathrm{~d}$ & $0 \mathrm{c}$ & $100 \mathrm{a}$ & $95 \mathrm{a}$ \\
\hline 12 & $2,4-D+$ picloran & 3,0 & $0 \mathrm{e}$ & $0 \mathrm{~d}$ & $0 \mathrm{c}$ & $100 \mathrm{a}$ & $100 \mathrm{a}$ \\
\hline 13 & $2,4-D$ amina + glifosato & $1,5+2,0$ & $0 \mathrm{e}$ & $0 \mathrm{~d}$ & $0 \mathrm{c}$ & $85 \mathrm{bc}$ & $20 \mathrm{c}$ \\
\hline 14 & $2,4-D$ amina + glifosato & $2,0+2,0$ & $0 \mathrm{e}$ & $0 \mathrm{~d}$ & $0 \mathrm{c}$ & $90 \mathrm{ab}$ & $25 \mathrm{c}$ \\
\hline 15 & lodosulfurom-metílico & 70 & $75 \mathrm{~d}$ & $65 \mathrm{c}$ & $90 \mathrm{ab}$ & $85 \mathrm{bc}$ & $90 \mathrm{a}$ \\
\hline 16 & lodosulfurom-metílico & 100 & $95 \mathrm{a}$ & $90 \mathrm{ab}$ & $100 \mathrm{a}$ & $98 \mathrm{a}$ & $100 \mathrm{a}$ \\
\hline 17 & Testemunha & -- & $0 \mathrm{e}$ & $0 \mathrm{~d}$ & $0 \mathrm{c}$ & $0 \mathrm{e}$ & $0 \mathrm{~d}$ \\
\hline
\end{tabular}

Médias seguidas pela mesma letra, na coluna, não diferem significativamente, pelo teste de Duncan, ao nível de $5 \%$ de probabilidade.

$\mathrm{Na}$ última avaliação de controle, realizada aos 40 DAT, observou-se que o melhor controle de aveia preta e de aveia branca foi obtido com os tratamentos clodinafop-propargil, dose acima de $100 \mathrm{~mL}$ p.c. ha ${ }^{-1}$, iodosulfurom-metílico, dose de 100 g p.c. ha ${ }^{-1}$, e diclofop-methyl, dose de 3,0 L p.c. ha ${ }^{-1}$ (Tabela 6).

Controle eficiente de azevém, acima de 95\%, foi obtido com diclofop-methyl, na dose de 1,5 L p.c. ha ${ }^{-1}$, com o clodinafop-propargil, na dose de $200 \mathrm{~mL}^{\text {p.c. }} \mathrm{ha}^{-1}$, e com iodosulfurom-metílico, na dose de $70 \mathrm{~g} \mathrm{p.c.} \mathrm{ha-1}$. O herbicida clodinafoppropargil, na dose de $100 \mathrm{~mL}$ p.c. $\mathrm{ha}^{-1}$, evidenciou controle intermediário de azevém, indicando a necessidade de uso de doses mais elevadas (Tabela 6). 0 
nabo foi controlado com eficiência pelos tratamentos metsulfuron-methyl, na dose de $6 \mathrm{~g}$ p.c. ha ${ }^{-1}$, iodosulfurom-metílico, na dose de $100 \mathrm{~g}$ p.c. ha ${ }^{-1}, 2,4-\mathrm{D}$, aplicado em pós-emergência, na dose de 1,0 L p.c. ha ${ }^{-1}$, e 2,4-D + picloram, na dose de 1,5 L p.c. $\mathrm{ha}^{-1}$. Os tratamentos iodosulfurom-metílico, na dose de $70 \mathrm{~g} \mathrm{p.c.} \mathrm{ha-}{ }^{-1}, 2,4-\mathrm{D}$, aplicado em pré-emergência, na dose de 1,5 e 2,0 L p.c. ha ${ }^{-1}$, e metsulfuron-methyl, na dose de $4 \mathrm{~g}$ p.c. ha $^{-1}$, apresentaram controle de nabo entre 80 e $85 \%$ (Tabela 6). A ervilhaca foi controlada com eficiência pelos tratamentos contendo iodosulfurommetílico, na dose de $70 \mathrm{~g}$ p.c. ha ${ }^{-1}, 2,4-\mathrm{D}$, aplicado em pós-emergência, na dose de 1,0 L p.c. ha ${ }^{-1}$, e 2,4-D + picloram, na dose de 1,5 L p.c. ha ${ }^{-1}$. Os tratamentos contendo bentazon, metsulfuron-methyl e 2,4-D, aplicado em pré-emergência, proporcionaram baixos níveis de controle de ervilhaca, entre 15 e 50\% (Tabela 6).

Existem relatos na literatura sobre a seletividade do herbicida diclofop-methyl ao trigo e à cevada e seu potencial de controle de outras espécies gramíneas, como aveia preta, aveia branca e azevém (Holt et al., 1993). Quanto aos demais herbicidas graminicidas avaliados, clodinafop-propagil e iodosulfurom-metílico, por serem moléculas recém-lançadas no mercado, as informações disponíveis são mais restritas.

Tabela 6. Avaliação da fitotoxicidade, aos 40 DAT, em aveia branca, em aveia preta, em nabo e em ervilhaca, em resposta à aplicação de diferentes herbicidas e doses. Embrapa Trigo-CNPT, Passo Fundo, RS, 2005.

\begin{tabular}{|r|c|r|r|r|r|r|r|}
\hline $\mathbf{N}^{\circ}$ & Herbicida & $\begin{array}{c}\text { Produto } \\
\text { comercial } \\
\text { (L ou g ha- }\end{array}$ & $\begin{array}{c}\text { Aveia } \\
\text { preta }\end{array}$ & $\begin{array}{c}\text { Aveia } \\
\text { branca }\end{array}$ & Azevém & Nabo & Ervilhaca \\
\hline 1 & bentazon & 1,5 & $0 \mathrm{c}$ & $0 \mathrm{c}$ & $0 \mathrm{c}$ & $50 \mathrm{c}$ & $35 \mathrm{~cd}$ \\
\hline 2 & bentazon & 2,5 & $0 \mathrm{c}$ & $0 \mathrm{c}$ & $0 \mathrm{c}$ & $65 \mathrm{c}$ & $50 \mathrm{~b}$ \\
\hline 3 & metsulfuron-methyl & 4 & $0 \mathrm{c}$ & $0 \mathrm{c}$ & $0 \mathrm{c}$ & $80 \mathrm{~b}$ & $25 \mathrm{de}$ \\
\hline 4 & metsulfuron-methyl & 6 & $0 \mathrm{c}$ & $0 \mathrm{c}$ & $0 \mathrm{c}$ & $90 \mathrm{ab}$ & $45 \mathrm{bc}$ \\
\hline 5 & diclofop-methyl & 1,5 & $85 \mathrm{~b}$ & $80 \mathrm{~b}$ & $95 \mathrm{a}$ & $0 \mathrm{~d}$ & $0 \mathrm{f}$ \\
\hline 6 & diclofop-methyl & 3,0 & $90 \mathrm{ab}$ & $85 \mathrm{ab}$ & $98 \mathrm{a}$ & $0 \mathrm{~d}$ & $0 \mathrm{f}$ \\
\hline 7 & clodinafop-propargil & 100 & $95 \mathrm{ab}$ & $90 \mathrm{ab}$ & $80 \mathrm{~b}$ & $0 \mathrm{~d}$ & $0 \mathrm{f}$ \\
\hline 8 & clodinafop-propargil & 200 & $98 \mathrm{a}$ & $95 \mathrm{a}$ & $95 \mathrm{a}$ & $0 \mathrm{~d}$ & $0 \mathrm{f}$ \\
\hline 9 & $2,4-D$ amina & 1,0 & $0 \mathrm{c}$ & $0 \mathrm{c}$ & $0 \mathrm{c}$ & $100 \mathrm{a}$ & $95 \mathrm{a}$ \\
\hline 10 & $2,4-D$ amina & 2,0 & $0 \mathrm{c}$ & $0 \mathrm{c}$ & $0 \mathrm{c}$ & $100 \mathrm{a}$ & $100 \mathrm{a}$ \\
\hline 11 & $2,4-D+$ picloran & 1,5 & $0 \mathrm{c}$ & $0 \mathrm{c}$ & $0 \mathrm{c}$ & $100 \mathrm{a}$ & $95 \mathrm{a}$ \\
\hline 12 & $2,4-D+$ picloran & 3,0 & $0 \mathrm{c}$ & $0 \mathrm{c}$ & $0 \mathrm{c}$ & $100 \mathrm{a}$ & $100 \mathrm{a}$ \\
\hline 13 & $2,4-D$ amina + glifosato & $1,5+2,0$ & $0 \mathrm{c}$ & $0 \mathrm{c}$ & $0 \mathrm{c}$ & $80 \mathrm{~b}$ & $15 \mathrm{e}$ \\
\hline 14 & $2,4-D$ amina + glifosato & $2,0+2,0$ & $0 \mathrm{c}$ & $0 \mathrm{c}$ & $0 \mathrm{c}$ & $85 \mathrm{~b}$ & $30 \mathrm{~cd}$ \\
\hline 15 & lodosulfurom-metílico & 70 & $85 \mathrm{~b}$ & $80 \mathrm{~b}$ & $93 \mathrm{a}$ & $85 \mathrm{~b}$ & $95 \mathrm{a}$ \\
\hline 16 & lodosulfurom-metílico & 100 & $95 \mathrm{ab}$ & $85 \mathrm{ab}$ & $100 \mathrm{a}$ & $98 \mathrm{a}$ & $100 \mathrm{a}$ \\
\hline 17 & Testemunha & -- & $0 \mathrm{c}$ & $0 \mathrm{c}$ & $0 \mathrm{c}$ & $0 \mathrm{~d}$ & $0 \mathrm{f}$ \\
\hline
\end{tabular}

Médias seguidas pela mesma letra, na coluna, não diferem significativamente, pelo teste de Duncan, ao nível de $5 \%$ de probabilidade.

Os resultados, de forma geral, indicam que os herbicidas, nas doses avaliadas, são seletivos para as culturas de trigo, de triticale e de centeio, e que a cevada apresenta maior sensibilidade aos herbicidas, principalmente a iodosulfurommetílico, indicando que a aplicação desse herbicida em cevada deve ser criteriosa. Evidenciam ainda que aveia preta, aveia branca e azevém apresentam resposta diferenciada aos herbicidas diclofop-methyl, clodinafop-propargil e iodosulfurommetílico. Como a dose é fator importante no nível de controle de cada espécie, as 
indicações formuladas devem ser específicas para cada uma delas. Por sua vez, nabo e ervilhaca são controlados com eficiência pelos herbicidas iodosulfurommetílico e 2,4-D e 2,4-D + picloram.

\section{BIBLIOGRAFIA CITADA}

BLANCO, H. E.; OLIVEIRA, D. A., ARAÚJO, J. B. M.; GRASSI, N. 1973. Observações sobre o período em que as plantas daninhas competem com a soja. $O$ Biológico, 39: 31-35.

GARCIA-TORRES, L. \& FERNANDES-QUINTANILLA, C. F. Fundamentos sobre mala hierbas y herbicidas. Madrid : Mundi Prensa, 1991, 348 p.

HOLT, J. S.; POWLES, J. B.; HOLTUM, A. M. Mechanism and agronomic aspects of herbicide resistance. Annual Review of Plant Physiology and Plant Molecular Biology. Palo Alto, v.44, p. 203-229, 1993.

SWANTON, C. J. \& WEISE, S. F. 1991. Integrated weed management: the rationale approach. Weed Technol. 5: 657-663. 